\title{
Proceedings of the Scottish Otolaryngological Society Meeting, 13 May 2011, Dunblane, Scotland, UK
}

Socioeconomic status and health-related quality of life in otolaryngology patients

\author{
L Fraser ${ }^{1}$, I R C Swan ${ }^{2}$ \\ From ${ }^{1}$ Gartnavel General Hospital and ${ }^{2}$ Glasgow Royal Infirmary, \\ Glasgow
}

\section{Objective}

To assess whether socioeconomic status influences the health-related quality of life of patients referred to otolaryngology services.

\section{Method}

We performed a prospective, multicentre survey of patients attending six otolaryngology departments across Scotland. Patients were invited to complete a Health Utilities Index Mark 3 questionnaire before and after treatment. Data on symptoms, diagnosis and treatment were collected. Each patient's postcode was used to generate their Carstairs deprivation score as a measure of deprivation in their area of residence.

\section{Results}

Initial data were collected from 9047 patients and follow-up data were obtained from 4924 patients.

The mean deprivation score of patients varied greatly between the six centres, from -2.1 (confidence interval (CI) -2.2 to -2.0 ) (least deprived) to 4.2 (CI 4.0-4.4) (most deprived).

Patients in the most deprived areas had significantly lower initial Health Utilities Index Mark 3 questionnaire scores (mean 0.57, CI 0.56-0.59) than those in more affluent areas (mean 0.71 , CI $0.70-0.72$ ), regardless of condition.

The change in questionnaire scores after treatment did not vary with deprivation score.

\section{Conclusion}

The mean Carstairs deprivation score of patients attending different Scottish otolaryngology departments varied widely. We found that higher deprivation scores were associated with lower health-related quality of life on initial presentation, regardless of the presenting condition, but did not influence the change in health-related quality of life with treatment.

Voice disorders among teachers: is there a need for a preventative programme?

S Ansari, F MacGregor

From Gartnavel General Hospital, Glasgow
Objectives

To characterise voice disorders among teachers, and to assess if there was a need for a preventative programme.

\section{Method}

Teachers attending the voice clinic at the Gartnavel General Hospital between July 2005 and June 2010 were identified. Their clinical notes were examined and they were mailed a questionnaire about their work pattern.

Results

A total of 108 teachers were identified. Of these, 17 per cent had vocal fold nodules and 66 per cent attended speech therapy. The postal questionnaire was completed by 53 patients ( 55 per cent). Twenty-five per cent had taken sick leave due to dysphonia (mean duration 37 days, range 3-120). One had taken early retirement. The largest groups were primary ( 41 per cent) and secondary ( 33 per cent) school teachers. Only 22 per cent worked in an open plan classroom, and just 10 per cent had access to a microphone. Over 90 per cent had received no voice care advice or support during training or in the workplace.

\section{Conclusion}

Teachers are at risk of significant dysphonia resulting in lost working days but receive very little advice or support, whether in an undergraduate or a postgraduate setting.

\section{Management of recurrent croup in children}

I Rankin, S M Wang, A Waters, W A Clement, H Kubba

From The Royal Hospital for Sick Children, Glasgow

\section{Objectives}

Recurrent croup is relatively common but little is known about its aetiology and prognosis. We performed a retrospective study to describe the outcome of investigation and management of these children.

\section{Methods}

Case records were reviewed for all children presenting with recurrent croup from November 2002 to March 2011. Specific data collected included sex, age of onset, age at presentation, duration of croup prior to referral, number of episodes, comorbidity, hospital admissions, investigations performed, treatments given, duration of follow up and status at last follow up.

Results

Ninety children with recurrent croup were identified. Twenty-five patients (28 per cent) had anatomical airway abnormalities, of whom 16 (18 per cent) demonstrated various degrees of subglottic stenosis. Twenty-three children 
(26 per cent) had microlaryngobronchoscopy findings of reflux; these children often responded well to anti-reflux medication.

\section{Conclusion}

Recurrent croup requires microlaryngobronchoscopy for diagnosis of airway anomalies and reflux laryngitis. Routine measurement of immunoglobulin $\mathrm{E}$ and complement proteins is unhelpful.

How we do it: hyaluronidase injection for the rhinoplasty patient

D Baring, J Marshall

From Southern General Hospital, Glasgow

\section{Introduction}

Septorhinoplasty patients undergo injection of local anaesthetic with adrenaline to reduce intra-operative bleeding. This creates a mass effect which alters the contour of the nose during surgery, and which persists even after massage.

Hyaluronidase (Hyalase ${ }^{\mathrm{TM}}$ ) reversibly binds to hyaluronic acid in the intercellular matrix, permitting greater diffusion of fluid through tissue. It is used in other specialties to achieve greater spread of anaesthetic and to reduce swelling.

\section{Methods}

After induction of general anaesthesia, 1500 IU hyaluronidase mixed with $10 \mathrm{ml}$ lignocaine 1 per cent with 1:200 000 adrenaline was injected into the nose. (During the presentation, this process was illustrated using photographs and a video.)

\section{Results}

There was no residual soft tissue distortion within 3 minutes of injection. No benefit was lost. The injection site was not critical, and could be varied as required.

\section{Discussion}

Cottle described the use of hyaluronidase in rhinoplasty in 1950. It has an excellent safety profile, being used by other specialties, and is available in most anaesthetic rooms.

We have found this technique beneficial in more than five hundred rhinoplasty operations. It is especially helpful for subtle nasal deformities where minor changes to the appearance of the nose are critical, e.g. supra-tip depression and nasal tip surgery.

\section{Paediatric midline swellings requiring surgical} excision: the Yorkhill experience

K Mitchell, F B MacGregor, H Kubba

From The Royal Hospital for Sick Children, Glasgow

\section{Objectives}

To examine the causes and investigation of paediatric midline neck swellings requiring surgical excision, and to assess post-operative results.

\section{Study design}

Retrospective examination of operation notes, imaging and histological reports.

\section{Methods}

We retrospectively studied 64 children with midline neck swellings surgically excised at Yorkhill (The Royal Hospital for Sick Children) between 2000 and 2010.
Results

The excised lesions comprised: 36 thyroglossal duct cysts, 23 dermoid cysts, two lymph nodes, two lesions including both a thyroglossal duct cyst and a dermoid cyst, one branchial sinus cyst, and one follicular thyroid carcinoma. The majority ( 89 per cent) of patients underwent ultrasound scanning prior to surgery. Only 22 (38.6 per cent) of the 64 ultrasound scans correctly predicted the underlying pathology. One patient with a thyroglossal duct cyst had a cystectomy but the lesion recurred. There were three thyroglossal duct cyst recurrences (3.1 per cent) in total, two in complex cases.

\section{Conclusion}

This study demonstrates a higher incidence of dermoid cysts than previously reported. Ultrasonography has a very limited diagnostic role, but is important in confirming the presence of a normal thyroid gland. Recurrence rates are low if the correct surgery is performed.

Dexamethasone to prevent post-operative nausea and vomiting in tonsillectomy patients: an audit of Scottish Intercollegiate Guidelines Network guideline 117

\section{F Hathorn, P Steele, M-L Montague}

From St John's Hospital, Livingston, and The Royal Hospital for Sick Children, Glasgow

\section{Introduction}

The Scottish Intercollegiate Guidelines Network guideline 117 recommends the use of dexamethasone to prevent post-operative nausea and vomiting in children undergoing tonsillectomy. This should also be considered in adults. The audit compares dexamethasone use in tonsillectomy patients with the 'gold standard' set by the Scottish Intercollegiate Guidelines Network.

\section{Methods}

Data were collected prospectively on tonsillectomy patients at St John's Hospital, Livingston, and The Royal Hospital for Sick Children, Edinburgh. These first audit results and guidelines were presented to the ENT surgeons and anaesthetists. A second audit was then performed, completing the audit loop.

\section{Results}

Sixty-nine per cent of adults received dexamethasone (35/ $51)$. Overall, 64 per cent of children received dexamethasone $(25 / 39), 78$ per cent at The Royal Hospital for Sick Children and 33 per cent at St John's Hospital. Post-intervention, 72 per cent $(26 / 36)$ of adults received dexamethasone. Post-intervention, 76 per cent $(13 / 17)$ of children received dexamethasone, 78 per cent at $\mathrm{St}$ John's Hospital and 76 per cent at The Royal Hospital for Sick Children.

\section{Discussion}

Raising the profile of the Scottish Intercollegiate Guidelines Network guideline 117 among ENT anaesthetists appeared to result in a higher rate of compliance with the recommendations, when managing children undergoing tonsillectomy at the non-specialist paediatric hospital. The guidelines raised concerns amongst anaesthetists, particularly with respect to the high dexamethasone doses recommended. 
Functional outcome of early glottic squamous cell carcinoma patients receiving primary treatment comprising endoscopic laser resection or radiation

C Y Sau, S Robertson, J C L Yeo, K MacKenzie

From Glasgow Royal Infirmary

\section{Background}

The aim of this study was to evaluate the functional voice and swallowing outcomes of patients with tumour stage (T) 1 glottic carcinoma receiving primary treatment comprising endoscopic laser resection or radiotherapy.

\section{Methods}

The West of Scotland Managed Clinical Network database was analysed. Thirty-seven patients with carcinoma in situ and $\mathrm{T}_{1}$ invasive squamous cell carcinoma treated with either laser surgery or radiotherapy completed three questionnaires each: the Voice Symptom Scale, the M D Anderson Dysphagia Inventory Questionnaire and the University of Washington Quality of Life Questionnaire. Answers were evaluated.

\section{Results}

Of the 37 patients, 16 (43 per cent) had endoscopic laser resection as their primary treatment and 21 (57 per cent) had radiotherapy. Self-reported Voice Symptom Scale scores were better in the radiotherapy cohort than the endoscopic laser resection cohort. Swallowing outcomes were similar for the two groups.

\section{Conclusion}

At the time of writing, the West of Scotland Managed Clinical Network database continued to incorporate new patients' details and to update existing patients' data. This study was performed at a preliminary stage, and found little significant difference in swallowing outcomes between the two treatment modalities studied.

\section{Auditing epistaxis: results of implementing a management protocol}

\author{
L Melia, S Lim, O J Hilmi, G W McGarry \\ From Glasgow Royal Infirmary
}

\section{Objective}

To audit epistaxis care within North Glasgow ENT services before and after implementation of an epistaxis management protocol.

\section{Methods}

We undertook retrospective audits of epistaxis patients managed from 1999 to 2004 and from 2007 to 2009. Key indices included length of in-patient episode, transfusion rate, treatment methods, operative salvage rate, and timing and nature of salvage procedure.

\section{Results}

In 2006, we presented an audit of epistaxis management that showed that an unacceptably high proportion of patients were being managed by junior members of the team, and were being subjected to repeated and ineffective packing. A new epistaxis management protocol was subsequently implemented. Following this, a second audit showed that: over 70 per cent of patients were managed initially with direct therapy; packing rates had fallen from 72 per cent to only 28 per cent; mean in-patient stay had decreased from
4 days to 1 day; and the transfusion rate had decreased from 2.5 per cent to 1 per cent. Both audits found that, following surgical intervention, final discharge was achieved within 24 hours.

\section{Discussion}

Epistaxis is the commonest ENT emergency in the UK. Direct therapy is considered to be best practice, in order to shorten the in-patient episode. The literature suggests that hospital stay, transfusion requirement and operative intervention rates are useful surrogate measures with which to monitor the quality of epistaxis management.

The Grampian vestibular clinic: findings over four years

N Jeffrey, M Shakeel, G McHardy, C Brewis

From Aberdeen Royal Infirmary

\section{Introduction}

Vestibular disorders affect a significant number of adults in the UK, a good proportion of whom are referred to an otolaryngologist for further assessment and rehabilitation. In the Grampian NHS Trust vestibular clinic, audiological scientists perform detailed vestibular assessment and arrange rehabilitation if required. Not everybody seen in the general otolaryngology clinic needs to be assessed in this specialised clinic; rather, only complicated cases should be referred by the otolaryngologist to this service.

\section{Aim}

To investigate the efficiency of utilisation of the vestibular clinic, by auditing the pattern of referrals, clinical examination findings and need for formal vestibular rehabilitation.

\section{Patients and methods}

We undertook retrospective data collection from a prospectively maintained, electronic audiology database containing reports of all vestibular assessments undertaken from 2006 to 2010. Data were collected on demographics, referring physician, assessing audiologist, vestibular assessment results and need for formal vestibular rehabilitation. The Excel and Statistical Package for the Social Sciences software programs were used for data collection and analysis.

Results

A total of 490 patients were referred to the vestibular clinic. Most referrals (62 per cent; $305 / 490$ ) were made by the consultant. Seventy-four per cent $(362 / 490)$ of patients were female and 26 per cent $(128 / 490)$ were male. Significant scores for anxiety, depression and hyperventilation were seen in 25,15 and 31 per cent of patients, respectively. Sixty per cent of patients had an abnormal audiometric result. Abnormal vestibular assessment results were seen in the following proportions of patients: Dix-Hallpike test, 6 per cent; caloric test, 28 per cent; Romberg's test, 15 per cent; and sharpened Romberg's test and Romberg's test on foam, 47 per cent. Based on their clinical history and vestibular assessment results, most patients were reassured, given written exercises and discharged back to their general practitioner's care. Formal rehabilitation was required for only 21 per cent $(105 / 490)$ of patients.

\section{Conclusion}

The majority of referrals for vestibular assessment were from consultants. Balance disorders were more prevalent amongst females. The majority of patients referred for vestibular assessment did not require vestibular rehabilitation. It 
would appear that patients referred by non-consultants were more likely to require formal vestibular rehabilitation than those referred by consultants (31 vs 15 per cent, respectively).

Role of patient-reported outcome measures in

N K F Koo Ng, G W McGarry

From Glasgow Royal Infirmary

\section{Background}

National Health Service cutbacks have resulted in an increasing need to demonstrate and prove the effectiveness of surgical procedures. The Sino-Nasal Outcome Test 22 questionnaire is a well proven rhinology patient-reported outcome measure.

Aim

To compare pre- and post-operative Sino-Nasal Outcome Test 22 questionnaire scores for general rhinological procedures, for patients managed between November 2008 and October 2010 at the Glasgow Royal Infirmary.

\section{Methods}

We performed a prospective study of consecutive adult patients undergoing functional endoscopic sinus surgery (FESS), septoplasty and septorhinoplasty. The Sino-Nasal Outcome Test 22 questionnaire was completed pre-operatively and again post-operatively; these two scores were compared.

\section{Results}

A total of 231 procedures were performed on 221 patients. Overall data completeness was 49 per cent. For FESS, the mean Sino-Nasal Outcome Test 22 questionnaire score was 46 pre-operatively and 22 post-operatively (within three months). For septoplasty, this same score was 46 pre-operatively and 17 post-operatively. For septorhinoplasty, the same score was 48 pre-operatively and 25 post-operatively.

\section{Conclusion}

There was a clinically significant improvement in mean SinoNasal Outcome Test 22 questionnaire scores (comparing preversus post-operative results) following FESS, septoplasty and septorhinoplasty. Data completeness is a problem for all studies of this type, including the English national patient-reported outcome measure programme. We advocate the universal use of patient-reported outcome measures to assess general rhinological procedures.

\section{Revision nasal surgery after septoplasty: trainees versus trainers}

T R Karlsson, M Shakeel, N Jeffrey, B Ram, K W Ah-See From Aberdeen Royal Infirmary
Objective

Septoplasty is a challenging operation to teach and learn. Otolaryngology trainees are expected to be able to successfully perform septoplasty at the beginning of their career. When assessing the success of any operation, one important parameter is the incidence of revision surgery. This study aimed to investigate the association between post-septoplasty revision nasal surgery incidence and primary surgeon grade.

\section{Methods}

We undertook a retrospective chart review of all patients who had undergone septoplasty with or without inferior turbinate reduction over a 12-year period (1998-2010) in a tertiary referral centre. Patients were identified from operating theatre log books, and were excluded if they had undergone any other nasal procedure (e.g. functional endoscopic sinus surgery). Data were collected on demographics, surgery type, surgeon grade and revision nasal surgery type. Microsoft Excel and the Statistical Package for the Social Sciences software programs were used for data collection and analysis.

Results

A total of 2168 eligible patients with a mean age of 39 years (range, 16-73) were investigated; 1519 were male (70.0 per cent) and 651 female ( 30.0 per cent).

Surgeons were subdivided into four categories: specialist registrar (grade one), senior house officer (grade two), staff grade surgeon (grade three) and consultant (grade four).

Two groups of patients were identified: group A, undergoing septoplasty alone (788 procedures, 36.3 per cent); and group B, undergoing septoplasty with inferior turbinate surgery (1380 procedures, 63.7 per cent).

Of the 788 procedures performed in group A, specialist registrars performed 499 (63.33 per cent), senior house officers 18 (2.28 per cent), staff grade surgeons 136 (17.26 per cent) and consultants 135 (17.13 per cent). The revision nasal surgery incidence in this group was 4.81 per cent for grade one surgeons compared with 5.19 per cent for grade four surgeons.

Of the 1380 procedures performed in group B, specialist registrars performed 857 (62.25 per cent), senior house officers 23 (1.67 per cent), staff grade surgeons 162 (11.74 per cent) and consultants 338 (24.49 per cent). The revision nasal surgery incidence in this group was 2.45 per cent for grade one surgeons compared with 1.78 per cent for grade four surgeons.

\section{Conclusion}

In our cohort, the overall revision nasal surgery incidence after septoplasty was comparable to the published literature. The revision rate was low in patients who underwent a concomitant inferior turbinate reduction. The grade of the surgeon did not appear to strongly influence the need for revision nasal surgery; thus, our patients did not appear to be disadvantaged if operated upon by trainees. 